\title{
Design and development of reinforced antibiotic eye drops in a community pharmacy
}

\begin{abstract}
Objective: Develop sterile vancomycin-amikacin reinforced antibiotics in smaller batches of finished products than those produced at the industrial level and under different working conditions. In this work, critical aspects concerning the elaboration process such as equipment and process evaluation, protocol development, physicochemical characterization, and microbiological tests were analyzed. In the first stage, the preparation of the formulations was carried out in the Department of Pharmaceutical Sciences of the Faculty of Chemical Sciences of the National University of Cordoba. In the second stage, the eye drops were prepared in the laboratory of Farmacia Vilarrubi, an office pharmacy authorized by the health authority and dedicated to the preparation of non-sterile compounding formulations. Both samples prepared at different manufacturing sites were subjected to the same physicochemical and microbiological tests.
\end{abstract}

Methods and results: The sample compounding formulations of Vancomycin, Amikacin or their combination, at a dose of $30 \mathrm{mg} / \mathrm{mL}$, were developed and subjected to physicochemical essays such as macroscopic observation, measurement of $\mathrm{pH}$, osmolality, particle size, and quantification of drug concentration, during 7 days stored under adequate conditions of light and temperature. In addition, microbiological assays were carried out to verify their sterility and procedure under aseptic conditions. All the formulations made of reinforced antibiotic eye drops demonstrated high physicochemical and microbiological stability during the evaluated period.

Conclusion: The reinforced antibiotic eye drops and the container used for their conservation showed correct sterility and microbiological stability during the time of use for the patient. It is possible to transfer small batch production of this type of quality sterile formulations to a pharmacy. For this, the training and education of the pharmaceutical professional is imperative.

Keywords: community pharmacy, sterile compounding, reinforced antibiotic, eye drops, stability

Abbreviations: Amk, amikacin; RAEDs, reinforced antibiotic eye drops; Vnc, Vancomicin; UNC, Universidad Nacional de Córdoba

\section{Introduction}

The advance in personalized drug therapy with formulas adjusted to the therapeutic needs of each patient not covered by industrial drug products has generated a breakthrough in community pharmacy. ${ }^{1}$ In these cases, pharmacists develop and prepare specific composition formulas for each patient's condition, an efficient and, in many cases, the only strategy to address the need for individualized therapies.

One of the great challenges in a community pharmacy is the development of sterile pharmaceutical formulations, since they must have equipment and facilities available to offer product safety, effectiveness, and reliability of the manufactured product. ${ }^{2}$ The availability of a laminar flow hood has become indispensable according to the concepts referred above. However, due to its high cost, this equipment is usually out of range of retail pharmacies and hospital pharmaceutical service.

In particular, the pharmacological treatment of ophthalmic diseases involves considerable complexities that, on many occasions, are technically infeasible for the pharmaceutical industry. For example, when marketed formulations containing classical antibiotics are not effective for ocular infections, reinforced antibiotic eye drops (RAEDs) are developed through compounding formulations. Such
Volume 9 Issue 6 - 202|

\author{
Carolina Del Valle Bessone,' María Laura \\ Ramirez,' Sofía Mickaela Martinez,' Silvina \\ Mabel Vilarrubi,,'2 Natalia Ángel Villegas,' \\ Daniel Alberto Allemandi,' Daniela Alejandra \\ Quinteros' \\ 'Unidad de Investigación y Desarrollo en Tecnología \\ Farmacéutica (UNITEFA), CONICET and Departamento \\ de Ciencias Farmacéuticas, Facultad de Ciencias Químicas, \\ Universidad Nacional de Córdoba, Ciudad Universitaria, \\ Argentina \\ ${ }^{2}$ Office Pharmacy Vilarrubi, Carlos Paz 336I, Córdoba, 5000, \\ Argentina
}

Correspondence: Daniela A Quinteros, PhD, Departamento de Ciencias Farmacéuticas, Facultad de Ciencias Químicas, Universidad Nacional de Córdoba, Ciudad Universitaria, 5000-Córdoba, Argentina, Tel/Fax +54-35I-5353865, Email danielaquinteros@unc.edu.ar,danielaaq@gmail.com

Received: November II, 202I | Published: December I4, 2021 formulations often have to go through a suitable process (fractioning, dilution, etc.) from solutions marketed for parenteral use to optimize personalized topical ocular drops. The sterile compounding formulations of RAEDs have been used mainly in the treatment of infectious pathologies in tissues located at the anterior segment of the eye. In intraocular infections (endophthalmitis), it is usually necessary to combine RAEDs with supplementary medication administered by other routes. ${ }^{3}$ The reformulations of RAEDs most frequently required at hospitals include combinations of vancomycin (Vnc)/ amikacin (Amk), Vnc/ceftazidime, and cefazolin/tobramycin. ${ }^{4-6}$ One of the main safety risks related to compounding formulations is the potential microbial contamination during the manufacturing process, particularly considering that antibacterial preservatives often cannot be used. On the other hand, relevant physicochemical properties are usually not optimized or/and evaluated, which may conspire against biopharmaceutical aspects such as intraocular penetration, irritation, and patient tolerance..$^{7-9}$

In this work, results from a comparative study related to RAEDs elaboration based on Amk sulfate and Vnc hydrochloride are reported, using two different processes, in conditions resembling those in the community pharmacy. Critical aspects concerning the elaboration process such as equipment and process evaluation, protocol development, physicochemical characterization, and microbiological tests were analyzed. Procedures established in this work promoted the stability of the formulation over time when elaborated in an office pharmacy. 


\section{Materials and methods}

\section{Preparation of RAEDs}

The compounding RAEDs were produced from commercial parenteral formulations of Amk sulfate $500 \mathrm{mg}$ (Northia ${ }^{\circledR}$, Argentina) and Vnc hydrochloride $500 \mathrm{mg}$ (Richet ${ }^{\circledR}$, Argentina).

Vnc, Amk, and Vnc-Amk RAEDs $(10 \mathrm{~mL})$ were prepared with a final concentration of $30 \mathrm{mg} / \mathrm{mL}$. For its preparation, a Vnc vial was initially reconstituted with $10 \mathrm{~mL}$ of phosphate buffer $\mathrm{pH}=6$ (Parafarm ${ }^{\circledR}$, Argentina). Using the same syringe, $6 \mathrm{~mL}$ of Vnc solution, $1.2 \mathrm{~mL}$ of Amk, and $\mathrm{NaCl}$ solution (Anedra ${ }^{\circledR}$, Argentina) (32 mg of $\mathrm{NaCl}$ dissolved in the minimum amount of buffer) were brought to the final volume of $10 \mathrm{~mL}$ with phosphate buffer $\mathrm{pH}=6$. The final formulation was filtered with $0.22 \mu \mathrm{m}$ sterilizing filters (Merck KGaA, Germany), and stored in a previously disinfected dropper bottle. The Amk, Vnc, and Vnc-Amk formulations were prepared in triplicate and stored at $8^{\circ} \mathrm{C}$.

The compounding of RAEDs was carried out following two different sterility protocols. First, a known specific protocol using a laminar flow hood (METHOD \#1) was used (School of Chemical Sciences, Universidad Nacional de Córdoba (UNC), Argentina). In the second method, the use of a laminar flow hood was omitted and the formulation was developed in a pretreated facility to create an aseptic environment (METHOD \#2). Thus, the operator cleans and disinfects the room, waiting 30 minutes to start the compounding process. In both methods, the materials (dropper bottle, syringe, buffer solution, filters) were processed conveniently to provide sterility.

\section{Physicochemical characterization and study of RAEDs stability}

The protocol for RAEDs manufacture was developed to comply with biopharmaceutical requirements for an ophthalmic formulation. Hence, a set of assays related to quality controls listed below were performed for 7 days. The samples were stored in preconditioned dropper bottles at temperatures between $2^{\circ} \mathrm{C}-8^{\circ} \mathrm{C}$ and protected from light.

\section{pH measurement}

A potentiometer (Seven Multi, Mettler Toledo, USA), calibrated at pH 4 and 7, was used for the measurement of Amk, Vnc, and VncAmk samples. Each sample was assayed in triplicate $(\mathrm{n}=3)$.

\section{Osmolality}

The osmolality test was carried out with an Osmometer (VAPRO, M5600, Wescor Inc., USA) using a $290 \mathrm{mmol} / \mathrm{kg}$ solution as a reference. The samples were tested in triplicate $(n=3)$. The initial osmolality of the samples was measured in phosphate buffer, and adjusted to physiological conditions (titled $\mathrm{NaCl}$ solution).

\section{Macroscopic observation}

Macroscopic observation was based on the determination of the absence and/or presence of particles, precipitate, or opalescence in the solutions. It was verified daily by visual examination, using a LED lamp and a black background.

\section{Determination of particle size}

To measure the size of contaminant particles, photonic correlation and micro-electrophoresis techniques were used (Delsa ${ }^{\mathrm{TM}}$ Nano Zeta, USA). Each sample was measured in triplicate $(n=3)$ at $25^{\circ} \mathrm{C}$. This technique was important to evaluate the stability of Vnc formulation as a function of $\mathrm{pH}$

\section{Qualitative/quantitative determination of $\mathrm{Vnc}$ and Amk}

The quantification of Vnc present in the Vnc and Vnc-Amk samples was carried out on an Agilent ${ }^{\circledR} 1100$ Series high-performance liquid chromatography (HPLC) equipped with an automatic injector, a column thermostat, a UV-Vis detector, and a column Phenomenex ${ }^{\circledR}$ $\mathrm{C} 185 \mu \mathrm{m}$ particle size, $15 \mathrm{~cm}$ longer $\mathrm{x} 0.4 \mathrm{~cm}$ internal diameter. The mobile phase was a buffer solution of $0.05 \mathrm{M}\left(\mathrm{NH}_{4}\right) 2 \mathrm{HPO}_{4}$ (Anedra ${ }^{\circledR}$, Argentina) at $\mathrm{pH}=4$ and acetonitrile (Sintorgan $\AA$, Argentina), in a ratio of $92: 8 \mathrm{v} / \mathrm{v}$. A flow of $1 \mathrm{~mL} / \mathrm{min}$ was used at a temperature of $40^{\circ} \mathrm{C}$, with UV detection at $220 \mathrm{~nm} .{ }^{10}$ The Amk concentration present in the Amk and Vnc-Amk samples was quantified by UV spectrophotometry (Evolution $^{\mathrm{TM}}$ 300, Fisher Scientific SL, Spain) by the derivatization method with o-Phthalaldehyde (OPA) (Sigma Aldrich, USA), in 1:1 ratio, at a wavelength of $340 \mathrm{~nm}$. A standard solution of $9 \mu \mathrm{g} / \mathrm{mL}$ concentration was used. The measurement was made two minutes following derivatization. To obtain the derivatization reagent, the methodology described by Caturla et al was followed. ${ }^{8}$

\section{Microbiological tests}

Two different culture mediums were used to perform the microbiological tests: Thioglycollate fluid medium (Britania ${ }^{\circledR}$, Argentina) for the detection of microorganisms (according to USP pharmacopoeia) and Tryptin Soy Agar $\left(\right.$ Britania $^{\circledR}$, Argentina) for the detection of fungi. Before use, their growth promotion capacity was verified. As positive control, thioglycollate broth inoculated with Staphylococcusaureus (ATCC No. 6538), Pseudomonasaeruginosa (ATCC No. 9027) and Clostridiumsporogenes (ATCC No. 11437) and Tryptin Soy broth inoculated with Bacillus subtilis (ATCC No. 6633), Candida albicans (ATCC No. 10231) and Aspergillus niger (ATCC No. 16404) were used. Both media were incubated for 5 days, while a non-inoculated medium grown under the same conditions was used as a negative reference.

\section{Evaluation of the aseptic technique and sterility test}

Vnc-Amk-RAEDs (10 mL, sample A) and the vehicle without the actives $(10 \mathrm{~mL}$, sample $\mathrm{B})$ were subjected to sterility assays in order to verify lack of contamination attributable to the methods of manufacture proposed. Samples processed according to METHOD \#1 were designated Samples A1 and B1. On the other hand, samples processed according to METHOD \#2 were named Samples A2 and B2 (Table 1).

Table I Classification of the samples to be measured

\begin{tabular}{llll}
\hline \multicolumn{2}{c}{ Sample } & Method & Composition \\
\hline A & Al & Laminar flow Hood (UNC) & $\begin{array}{l}\text { Amikacin-Vancomycin + } \\
\text { vehicle }\end{array}$ \\
& A2 & Office Pharmacy Laboratory & \\
& B & Laminar flow Hood (UNC) & Vehicle \\
& B2 & Office Pharmacy Laboratory &
\end{tabular}

Before and after sterilizing filtration, $1 \mathrm{~mL}$ of samples A1, A2, $\mathrm{B} 1$, and B2 was withdrawn from each container, carefully seeded in petri dishes with Tryptin soy Agar and incubated at $37^{\circ} \mathrm{C}$ for 24 hours for subsequent count of viable aerobes (Figure 1.I). The assays were performed in duplicate. The sterilization procedure was carried out following the membrane filtration method codified in the Argentine Pharmacopoeia. ${ }^{11}$ 
Initially, the formulations were filtered with a $0.22 \mu \mathrm{m}$ pore size filter device that was placed on the syringe loaded with the formulation. Then, another device with a $0.45 \mu \mathrm{m}$ pore size membrane was incorporated. Finally, the membranes were placed in Erlenmeyer flasks containing the culture broths (Thioglycollate broth and Tryptin soy broth) to observe growth. They were incubated in an oven at $37^{\circ} \mathrm{C}$ and $25^{\circ} \mathrm{C}$, respectively, and were observed 4 and 7 days later. Previously, the device was assembled and sterilized with the membrane placed (Figure 1.II).

\section{Microbiological stability essay}

The samples under test (Figure 1.III) were packed in preconditioned dropper bottles and stored in a temperature controlled environment (2$8^{\circ} \mathrm{C}$ ). The tests were carried out at $\mathrm{T}=0$ and $\mathrm{T}=7$ days to evaluate the behavior of the eye drops in its final container during the time studied. Aliquots of $1 \mathrm{~mL}$ were extracted from their containers, carefully seeded in Petri dishes containing Tryptin Soy Agar $(n=2)$ and they were incubated at $37^{\circ} \mathrm{C}$. In both cases, the presence of bacterial development was evaluated.

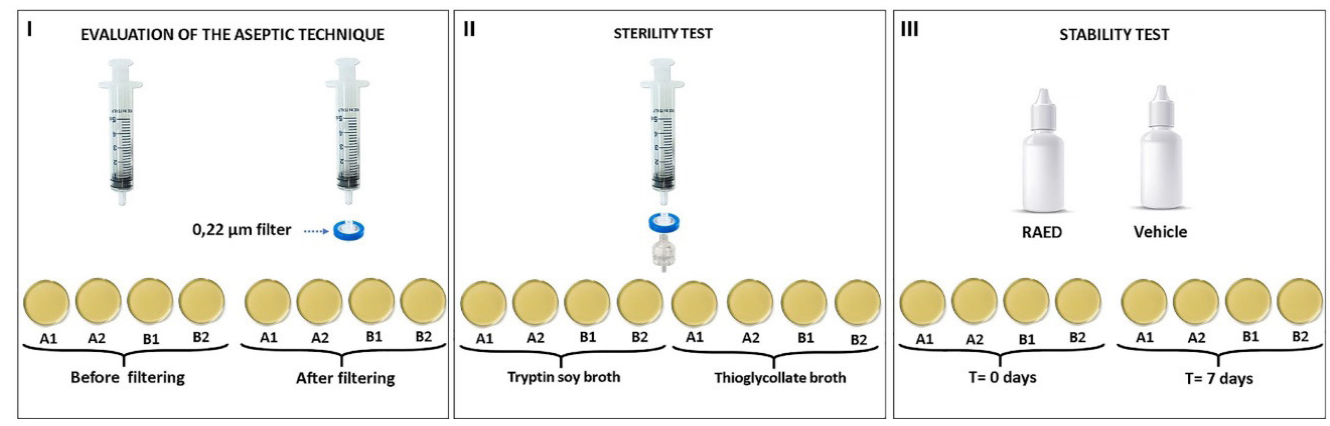

Figure I Methodology used for the (I) evaluation of the aseptic technique, before and after filtering, (II) sterility test in two different broths, and (III) stability test at 0 and 7 days of the test formulations.

\section{Results}

\section{Characterization and study of RAEDs sability of Vnc, Amk, and Vnc-Amk}

As for stability indicators, the $\mathrm{pH}$, osmolality and particulate content were evaluated in this work. Since the treatment of the most frequent ophthalmic pathologies usually lasts 7 days, stability studies were performed during this period. The results showed that the $\mathrm{pH}$ of the Vnc (5.62 \pm 0.01$)$, Amk (6.00 \pm 0.01$)$, and Vnc-Amk (5.61 \pm 0.01$)$ solutions were slightly more acidic than optimal for tear fluid $(\mathrm{pH}$ 6.6 to 7.8). Previous studies have shown that with the administration of ocular solutions with a $\mathrm{pH}$ between $5-6$, the general $\mathrm{pH}$ of the tear fluid lays to quickly reestablish itself to physiological values, causing minimal discomfort on the ocular surface. For this reason, it is expected that the application per se of the formulations will not cause irritating effects. ${ }^{12,13}$ Likewise, no variations in $\mathrm{pH}$ values were observed during this period of time (Figure 2.a).

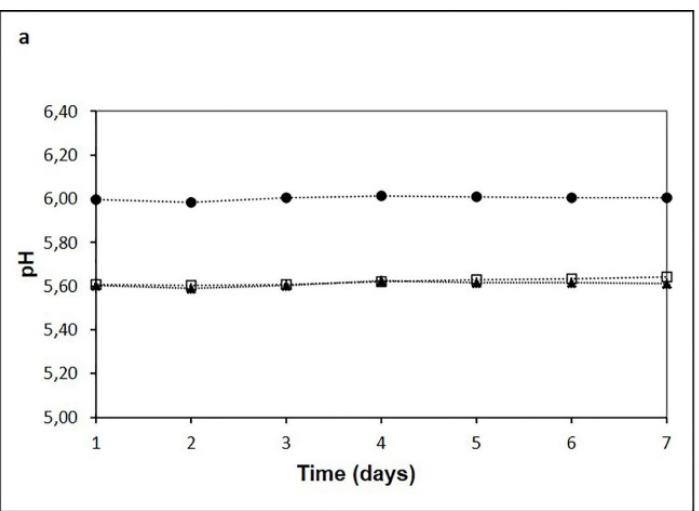

The osmolality of Amk (296.67 \pm 4.73$) \quad \mathrm{mmol} / \mathrm{Kg}, \quad \mathrm{Vnc}$ $(287.33 \pm 4.51) \mathrm{mmol} / \mathrm{Kg}$, and Vnc-Amk $(315.00 \pm 6.56) \mathrm{mmol} / \mathrm{Kg}$ formulations are close to that of the tear fluid. Based on these results, no eye irritation is expected, at least during the 7-day treatment. In addition, macroscopic observation did not show presence of precipitate, opalescence, color change, or suspended particles in the formulation. Results suggest that the method of obtaining is appropriate for the elaboration of ophthalmic formulations since it does not produce physical instabilities in the final formulation. ${ }^{14}$

Stability is also conditioned by properties such as particle size. Vnc-Amk formulation presented a particle size between 3-4 nm with a prevalence percentage of $96 \%$ (Figure 2.b), as did the Vnc formulation. Amk formulation contains particles smaller than 2.5 $\mathrm{nm}$ with a prevalence percentage greater than $80 \%$. The absence of agglomerations and/or increase in particle size as a function of time allows corroborating the results obtained from the macroscopic observation of RAEDs. Thus, the process of manufacture meets the safety criteria of freedom from extraneous foreign particulate matter in the ophthalmic formulations. ${ }^{15}$

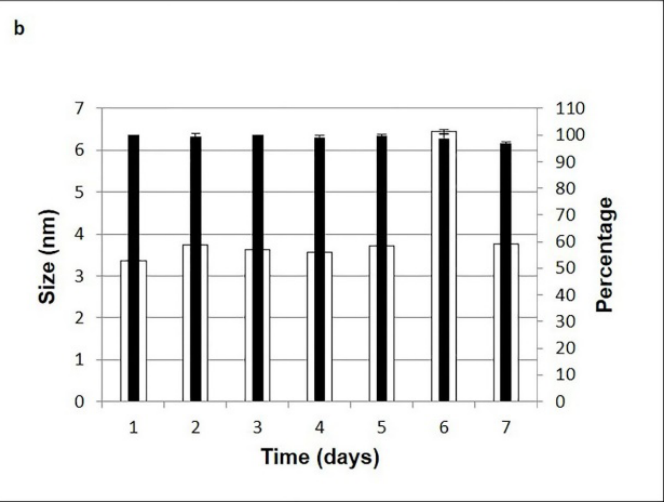

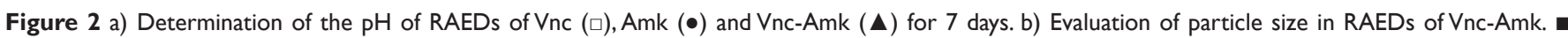
Percentage $\square$ Particle size. 


\section{Evaluation of the concentration of Vnc, Amk, and Vnc- Amk in the formulation}

The stability period of a pharmaceutical dosage form was determined as the time in which the remaining concentration of the active ingredient is equal to or higher than $90 \%$ of the initial concentration. Regarding drug content, USP N³1 considers that it should be between $90 \%-115 \%$ of the initial concentration. ${ }^{16}$ The concentration of each antibiotic was determined individually and associated. In both cases, the results obtained at time zero (day of preparation of solution) were considered equivalent to $100 \%$ of the concentration. Figure 3, shows the chemical stability curve $(\%$, expressed as residual concentration) of the mean values of Vnc and Amk $30 \mathrm{mg} / \mathrm{mL}$ solutions.
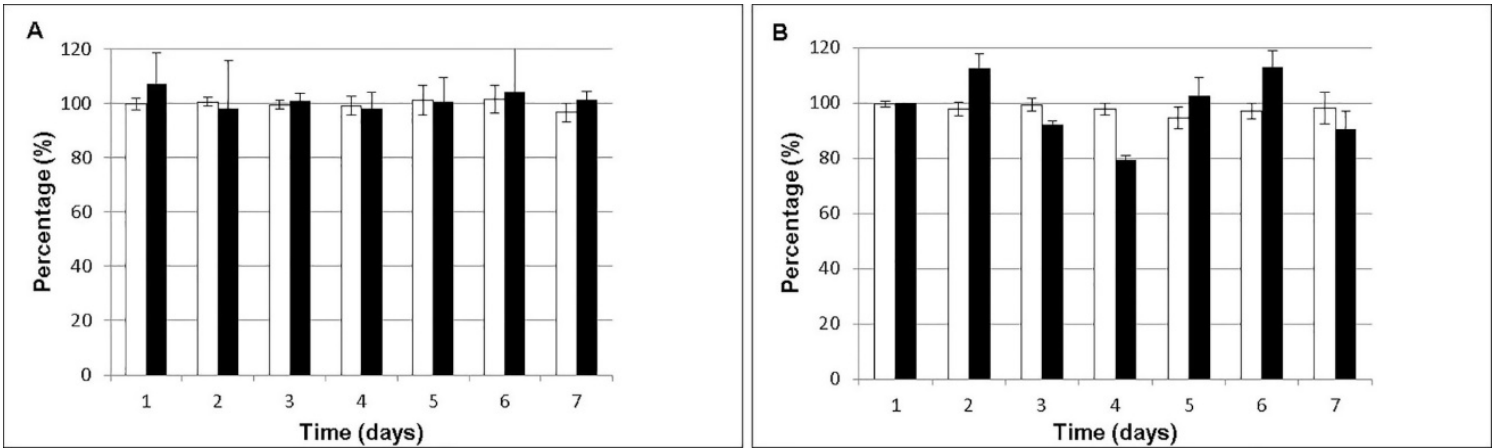

Figure 3 Stability (\%, residual concentration) of sample (A) and associated RAEDs (B). ( $\square$ Vnc, $\square$ Amk).

\section{Microbiological and sterility studies of RAEDs}

Sterility tests were performed according to Argentine Pharmacopeia. ${ }^{11}$ The aseptic technique of the test formulations with a lighter was evaluated in the community pharmacy and in the laminar flow hood. The results obtained for Vnc-Amk RAEDs (A) were compared to the vehicle $(\mathrm{NaCl}$, phosphate buffer $\mathrm{pH}=6)(\mathrm{B})$. Microbial growth was not observed in samples A and B. Thus, sterility requirements were fulfilled for both methods and all formulations. The efficacy of sterility procedures was verified by the lack of microbial growth in the culture media handled in the compounding environment. It was also evident that the RAEDs container sterilization process was efficient. ${ }^{17}$

\section{Discussion}

From the pharmaceutical point of view, the stability of the active ingredients is one of the most important criteria to determine the acceptance or rejection of any medication, particularly those administered by ophthalmic or parenteral routes. Stability studies are performed to determine the time and storage conditions in which raw materials and official preparations are kept within the specifications on identity, potency, quality, and purity. ${ }^{9}$ The stability of pharmaceutical products, in their final primary packaging, must be demonstrated using appropriate methods. A significant parameter to take into account is the $\mathrm{pH}$ of the formulation; the physiological $\mathrm{pH}$ of the tear fluid ranges from 6.5 to $7.6,{ }^{7}$ and the external surface of the eye tolerates preparations with a $\mathrm{pH}$ between 3.5 and $10.5^{2,18,19}$ However, solutions with $\mathrm{pH}$ below 6.5 or above 8.5 can cause corneal damage if contact is prolonged enough. ${ }^{20}$ Although the $\mathrm{pH}$ of our formulations was slightly acidic, taking into account that the estimated time of use of the formulations for pathologies such as keratitis and/or conjunctivitis is 7 days, the established $\mathrm{pH}$ range would not generate any type of damage. Guiu Segura et. al. evidenced that the maximum stability $\mathrm{pH}$ for $\mathrm{Vnc}$ is between 3 and $5 .{ }^{21}$ The $\mathrm{pH}$ of Vnc and Vnc-Amk RAEDs were (5.62 \pm 0.01$)$ and (5.61 \pm 0.01$)$, respectively. The values are slightly above this range; hence, their stability could be favored. Concerning osmolality, topical eye drops need to be isotonic ${ }^{22}$ like tear fluids, whose values range between 280 $293 \mathrm{mmol} / \mathrm{kg}$. Prolonged contact with hypotonic eye drops can cause corneal edema, while hypertonic eye drops can be painful during instillation due to their dehydrating effect on the corneal epithelium. ${ }^{21}$ Solutions with an osmolality fewer than $100 \mathrm{mmol} / \mathrm{kg}$ or greater than $640 \mathrm{mmol} / \mathrm{kg}$ cause eye irritation. ${ }^{23}$ The test formulations showed accepted osmolality values ranging from $280-315 \mathrm{mmol} / \mathrm{kg}$. Anna Kodym et al., have demonstrated similar $\mathrm{pH}$ and osmolarity values for ophthalmic formulations containing cefuroxime, a cephalosporin of the second generation. ${ }^{24}$ According to Pharmacopoeia, for smallvolume injectable solutions, the presence of particles with sizes greater than $10 \mu \mathrm{m}$ and $25 \mu \mathrm{m}$ indicates that the environment is not suitable for the analysis of particles. ${ }^{25}$ Results show the presence of very small particles, in the order of 2-4 nanometers at all studied times.

In respect of the values obtained from concentration of Vnc, Amk, and Vnc-Amk in the formulation, similar results have been reported by Alonso et al, where the physicochemical stability of an Amk solution $(25 \mathrm{mg} / \mathrm{mL}$ in $\mathrm{NaCl} 0.9 \% \mathrm{w} / \mathrm{v})$ stored at $2^{\circ} \mathrm{C}-8^{\circ} \mathrm{C}$ during 10 days was corroborated. ${ }^{26}$ Chiang et al. evaluated the stability of Vnc-Amk eye drops prepared from parenteral formulations with a concentration of Amk $20 \mathrm{mg} / \mathrm{mL}$ and Vnc $50 \mathrm{mg} / \mathrm{mL}$. These eye drops were kept at $4^{\circ} \mathrm{C}$, remaining stable for at least 14 days. ${ }^{27}$

Regarding microbiological tests, there are no clear regulatory requirements about the preparation of sterile dosage forms in community pharmacy laboratories. Current regulations are mainly applicable to the pharmaceutical industry. ${ }^{11,28,29}$ To guarantee the sterility of a formulation in pharmacy laboratories, the quality of the raw material, the manufacturing procedures, operator's skill, and the environmental conditions in which the procedure is performed must be considered. Another important factor involves the inclusion or exclusion of a preservative in ophthalmic solutions. ${ }^{30}$ It was possible to evaluate the feasibility of producing RAEDs without preservatives in a community pharmacy under lighters (aseptic conditions, METHOD \#2), and to compare the results obtained with the same eye drops produced in a laminar flow booth (UNC, METHOD \#1), very auspicious results were obtained. Although an utterly sterile working environment cannot be achieved, procedures such as disinfection of laboratory surfaces play a significant role in creating an aseptic environment. It is important to limit working exposure to drafts, avoiding contact of sterile instruments with non-sterile surfaces, 
and performing the procedures in an orderly manner, which greatly reduces formulation contamination. Staff education and training are the most important requirements to achieve desired results when the ideal equipment needed are not available in the community pharmacy.

\section{Conclusion}

In conclusion, the formulations proposed in our research work are simple and were made with minimum ingredients, without preservatives or excipients. The results show that the aseptic conditions in which the formulations of the compounds were carried out implemented with good manufacturing practices, stored with protection from light and specific temperatures, are correct. he results showed the RAED formulations and the container used for their conservation, showed correct sterility and microbiological stability during preparation and the estimated time of use by the patient.

\section{Credit taxonomy}

Carolina Del Valle Bessone: Conceptualization, Formal Analysis, Resources, Writing -Original Draft.

María Laura Ramirez: Methodology, Resources, Writing - Original Draft, Visualization.

Sofía Mickaela Martinez: Methodology, Resources, Writing Original Draft, Visualization.

Silvina Mabel Vilarrubi: Methodology, Validation, Resources.

Daniel Alberto Allemandi: Supervision and Editing.

Daniela Alejandra Quinteros: Supervision, Writing- Reviewing and Editing.

\section{Funding information}

The authors wish to acknowledge Consejo Nacional de Investigaciones Científicas y Técnicas (CONICET), Universidad Nacional de Córdoba and Laboratory of Blood Products (Laboratorio de Hemoderivados ${ }^{\circledR}$ ), Universidad Nacional de Córdoba, for financial support and facilities.

\section{Conflicts of interest}

The authors have no commercial or proprietary interests or conflicts of interest related to this submission.

\section{References}

1. Corral-Aragón A. Pharmaceutical Compounding in Ophthalmology: a Therapeutic Need. Arch Soc Esp Oftalmol. 2006;81:631-632.

2. Allen LJ. Basics of Sterile Compounding: Ophthalmic Preparations, Part 1: Ophthalmic Solutions. Int J Pharm Compd. 2016;20(5):399-404.

3. Barberá Loustaunau E, Vázquez Castro F. Tratamientos tópicos oculares: revisión. Inf Ter Sist Nac Salud. 2009;33(3):80-87.

4. Lin JM, Tsai YY, Fu YL. The Fixed Combination of Fortified Vancomycin and Amikacin Ophthalmic Solution-VA Solution. Cornea. 2005;24(6):717-721.

5. Pigrau C. Oxazolidinonas y glucopéptidos. Enferm Infecc Microbiol Clin. 2003;21(3):157-165.

6. Pigrau C, Almirante B. Oxazolidinonas, glucopéptidos y lipopéptidos cíclicos. Enferm Infecc Microbiol Clin. 2009;27(4):236-246.

7. Thakur SS, Bai A, Chan D, et al. Ex vivo evaluation of the influence of $\mathrm{pH}$ on the ophthalmic safety, antibacterial efficacy and storage stability of povidone-iodine. Clin Exp Optom. 2020; cxo.13100.
8. Caturla MC, Cusido E, Westerlund D. High-performance liquid chromatography method for the determination of aminoglycosides based on automated pre-column derivatization with o-phthalaldehyde. $J$ Chromatogr A. 1992;593(1-2):69-72.

9. Yoshioka S, Stella VJ. Stability of Drugs and Dosage Forms. Boston: Kluwer Academic Publishers; 2002.

10. Jesús Valle MJ de, López FG, Navarro AS. Development and validation of an HPLC method for vancomycin and its application to a pharmacokinetic study. J Pharm Biomed Anal. 2008;48(3):835-839.

11. Ministerio de salud de la Nación. Farmacopea Argentina. 7 ed; 2003.

12. Linda A. Felton P. Remington Essentials of Pharmaceutics. First edit. Edited by Linda A. Felton P, editor. Pharmaceutical Press, UK. London, UK: Pharmaceutical Press; 2013. 763 p.

13. Saettone MF, Bucci M, Speiser P. Ophthalmic Drug Delivery Biopharmaceutical, Technological and Clinical Aspects. FIDIA RESE. Speiser MFSMBP, editor. Springer Science+ Business Media, LLC; 1987.

14. Mazet R, Yaméogo JBG, Wouessidjewe D, et al. Recent advances in the design of topical ophthalmic delivery systems in the treatment of ocular surface inflammation and their biopharmaceutical evaluation. Pharmaceutics. 2020;12(6):570.

15. Ali Y, Lehmussaari K. Industrial perspective in ocular drug delivery. $A d v$ Drug Deliv Rev. 2006;58(11):1258-1268.

16. United States Pharmacopeial Convention. Farmacopea de los Estados Unidos de América. 31 ed. Formulario Nacional. 26 ed. Rockville [EEUU] : U.S. Pharmacopeial Convention I, editor; 2007.

17. Manish K, Kulkarni GT. Recent advances in ophthalmic drug delivery system. Int J Pharm Pharm Sci. 2012;4(1):387-394.

18. Punín-Crespo E, Ballester-Viéitez A, Dávila-Pousa C, et al. Aspectos Prácticos de la Farmacotecnia en un Servicio de Farmacia: Situación Actual. Master Line \& Prodigio S., editor. Astellas Pharma S.A; 2011.

19. Lim LT, Ah-Kee EY, Collins CE. Common eye drops and their implications for $\mathrm{pH}$ measurements in the management of chemical eye injuries. Int $J$ Ophthalmol. 2014;7(6):1067-1068.

20. Ahuja M, Dhake AS, Sharma SK, et al. Topical Ocular Delivery of NSAIDs. AAPS J. 2008;10(2):229-241.

21. Guiu Segura JM, Munné García M, Jiménez Lozano I, et al. Controles de calidad en la preparación de colirios antiinfecciosos extemporáneos preparados en un servicio de farmacia. In: 54 Congreso Nacional de la Sociedad Española de Farmacia Hospitalaria; 2009. 181 p.

22. Fialho SL, da Silva-Cunha A. New vehicle based on a microemulsion for topical ocular administration of dexamethasone. Clin Exp Ophthalmol. 2004;32(6):626-632.

23. Bessone CDV, Martinez SM, Luna JD, et al. Neuroprotective effect of melatonin loaded in ethylcellulose nanoparticles applied topically in a retinal degeneration model in rabbits. Exp Eye Res. 2020;200:108222.

24. Kodym A, Zawisza T, Taberska J, et al. Pharmaceutical technology physicochemical and microbiological properties of eye drops containing cefuroxime. Acta Pol Pharm. 2006;63(4):293-299.

25. Administración Nacional de Medicamentos A y TM. 1130. Validación De Métodos Analíticos. Farm Argentina Vol I. 2003;447-50.

26. Alonso-Herreros JM. Preparación de Medicamentos y Formulación Magistral para Oftalmología. Ediciones Díaz de Santos SA, editor. Madrid; 2003. 127 p.

27. Chiang CC, Lin JM, Chen WL, et al. Comparison of topical fixedcombination fortified vancomycin-amikacin (VA solution) to conventional separate therapy in the treatment of bacterial corneal ulcer. Eye. 2009;23(2):294-298. 
28. World Health Organization. Annex 6 WHO good manufacturing practices for sterile pharmaceutical products. WHO Technical Report Series, No. $961 ; 2011$.

29. Guía de Normas de Correcta Fabricación. Agencia Española de Medicamentos y Productos Sanitarios; 2020
30. Baudouin C, Labbé A, Liang H, et al. Preservatives in eyedrops: The good, the bad and the ugly. Prog Retin Eye Res. 2010;29(4):312-334. 